\title{
同相牵引供电系统的四重化结构改良及 控制算法优化方法
}

\author{
成庶, 刘畅, 唐建湘, 于天剑, 李凯迪
}

(中南大学 交通运输工程学院, 湖南 长沙 417000)

\begin{abstract}
摘 要: 同相供电是解决传统牵引供电系统及单重同相供电方案存在的过电分相、电能质量欠佳及结 构、控制算法缺陷等技术瓶颈的关键技术之一, 是实现电气化铁路朝着安全、高速、重载方向发展的必 然途径。在单重同相供电技术的基础上,从系统结构改进和控制算法优化两方面入手,通过融合电力 系统柔性输电并网及多重化逆变等技术,提出负序消除以及无功、谐波抑制能力更优的新型四重化同 相供电方案。最后, 设计并搭建出新型同相牵引供电系统的仿真模型, 通过一系列仿真试验对所提出 的方法进行了验证, 并取得了预期的效果。
\end{abstract}

\section{关 键 词: 同相牵引供电; 多重化逆变; 潮流控制; 电能质量}

\section{中图分类号: U224.8}

文章编号: 1000-2758(2020)05-1112-10
进入 21 世纪以来, 在中国高铁技术飞速发展和 日本、欧洲传统铁路强国技术革新的共同作用下, 以 高速、重载为代表的电气化铁路迎来了又一个发展 热潮。但随着电气化铁路朝着安全、高速、重载的趋 势发展,传统牵引供电系统中的无功、谐波、负序等 电能质量问题和 “过电分相” 等结构安全问题也变 得愈发严重 ${ }^{[1]}$, 安全、经济、稳定的电能供应标准愈 发突显出牵引供电系统在电气化铁路中的重要地 位, 其性能的优劣与否直接影响了铁路的实际运营 效能。如何发展新型牵引供电技术来解决传统供电 系统中亟待解决的诸多弊端、实现电气化铁路的技 术革新成为了当前众多业内科研工作者的首要研究 方向。

传统既有线上采用轮换相序的异相供电制式, 即电力系统的三相交流电按区间轮流为牵引网供 电, 其实质上是一种 “区段离散” 的单相供电。在此 基础上有学者提出采用 Scott、等平衡接线变压器作 为改进措施 ${ }^{[2-3]}$, 但理论研究发现, 机车负载在时空 分布上的随机性极大地限制了该方案的实际运行效 能 ${ }^{[3]}$ 。随着电力电子技术的发展, 基于无功、谐波 补偿来消除负序、改善电能质量的同相供电技术在
电气化铁路领域大放异彩, 其核心装置按技术原理 可分为 3 大类: 第一类采用有源电力滤波器 $\mathrm{APF}$ (active power filter), 张秀峰等 ${ }^{[4]}$ 提出一种基于单台 $\mathrm{YN}, \mathrm{d} 11$ 牵引变压器与三相四桥臂结构的有源滤波 器相结合的同相供电方案, 但其使用的补偿电流及 解耦控制算法的精度易受电流滞环及基波有功电流 反馈参数的影响; 第二类采用无功补偿装置 (STAT$\mathrm{COM}$ ), 彭露等 ${ }^{[5]}$ 总结了多种技术较为成熟、实际应 用也十分广泛的动态无功补偿方案。但统计结果表 明, 无功补偿方案存在着功率因数不高、系统结构复 杂等缺陷; 第三类为统一潮流控制器 UPFC ( unified power flow controller), 杨博等 ${ }^{[6]}$ 在移相 $\operatorname{SPWM}$ ( sinusoidal pulse width modulation) 多电平逆变电路的基 础上提出了解决电力系统三相负荷不平衡、无功谐 波污染问题的直接电流控制方案。但系统闭环控制 时无功补偿指令电流的响应迟滞, 易影响控制算法 的精度。庞棋峰 ${ }^{[7]}$ 对单重同相供电装置的可行性 做了试验验证,但该系统在控制策略及整体结构上 仍存在很大的改进空间。刘婷婷 ${ }^{[8]}$ 提出一种能够 实现三相/单相直接变换的仿德式贯通供电拓扑结 构, 但这种供电模式并不适合我国的铁路发展现状。 
王荣华等 ${ }^{[9]}$ 提出一种融合有源变流器与无源滤波 器的统一功率控制系统 UPCS ( unified power control system)，该基波补偿系统克服了传统有源补偿装置 容量大、成本高的缺陷, 有效改善了电网侧的电能质 量。但 UPCS 在有源变流器部分仅采用了基于幅相 控制的简易开环控制算法, 降低了系统应用的灵活 性和鲁棒性。

结合上述同相牵引供电系统中对电能质量改善 技术的研究现状分析可知, 当前同相牵引供电方案 的研究大多立足于电力变流装置的干扰隔离性能, 以及其附带的滤波、隔离器件的控制算法优化和拓 扑结构改进 2 个主要方面。宋平岗等 ${ }^{[10]}$ 在模块化 多电平换流器 MMC ( modular multilevel converters) 结构的铁路功率调节器 RPC ( railway power regulator) 基础上, 提出一种比 PIR 控制器更优的 PCI 比例复数积分控制算法, 进一步提升了系统的 电能质量改善性能,但该算法中参数计算过程复杂, 不适于实际运用推广。窦祥等 ${ }^{[11]}$ 提出采用均衡电 感复合的并联八重化移相 SPWM 逆变技术, 在克服 传统功率场效应管容量不足缺陷的同时, 还有效降 低了大容量 IGBT( insulated gate bipolar transistor) 开 关管的工作频率标准, 但外加多组复合电感一方面 会使电路复杂化, 另一方面会造成多余的负载损耗, 提高了方案成本。周洪霞等 ${ }^{[12]}$ 进行了多重化逆变 技术在电能质量改善方面的探究工作, 对本文的多 重化结构改进具有一定的启发作用。

针对当前世界范围内高速、重载电气化铁路快 速发展的时代背景以及国内新、旧汼引供电系统更 新换代之际对技术革新的迫切需求, 本文在分析和 对比现有技术方案的基础上, 将变流装置的控制算 法优化同系统拓扑结构的创新相融合, 提出了一种 基于四重化统一潮流控制技术的新型同相牵引供电 方案。本方案选用当前综合性能和经济效益最优的 统一潮流控制技术作为新型同相供电方案的技术基 础, 基于对同相供电技术方案的深人剖析,一方面对 同相供电装置的控制算法进行深度优化, 提高新型 系统的灵活适用性和抗干扰能力, 解决当前同相供 电方案存在的通用性低、鲁棒性弱等问题; 另一方面 通过引人多重化逆变技术 ${ }^{[14-15]}$, 提出电能质量改善 性能更优的四重化结构, 改善和提升现有单重装置 抑制无功及谐波的性能。

此外, 本文还针对当前研究大多局限于变流装 置子模块量级或简化铁路负载分析方法的不足, 在
结合实际工程线路参数的基础上搭建了集供电、变 电、输电及用电为一体的完整牵引供电系统仿真模 型。通过与现有单重装置的仿真实验结果对比分 析, 从全局层面凸显出本新型同相供电装置在电能 质量改善及同相供电性能方面的优越性和实际工程 价值。

\section{1 同相供电技术原理分析}

\section{1 同相供电装置的结构组成}

为实现电能质量优化和安全运营的目标, 推动 电气化铁路向着高速、重载、安全的方向发展, 早期 铁路从业者提出了异相轮换供电、采用平衡接线变 压器等效果不佳的折中方案, 其结构如图 1a) 所示。 但理论研究和工程实践效果反馈表明, 这类早期的 谐波、负序治理方案存在着诸多的弊端和应用条件 上的局限性 ${ }^{[3]}$ 。

随着大功率电力电子设备的推广, 以及柔性供 电技术在电力系统高压并网领域的成功应用, 发展 同相牵引供电技术成为了突破传统技术瓶颈的必然 选择。

区别于传统异相轮换供电方案, 同相牵引变电 所将平衡接线变压器与同相供电装置进行有机结 合, 能够实现对机车的贯通单相供电, 即在满足电能 供应质量标准的前提下取消了 “电分相区”, 如图 $1 b)$ 所示。

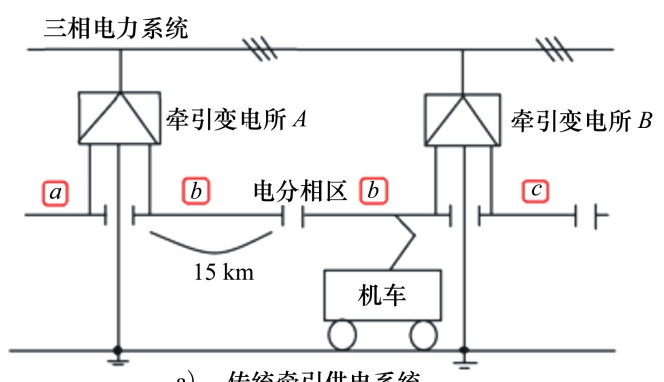

a）传统牵引供电系统

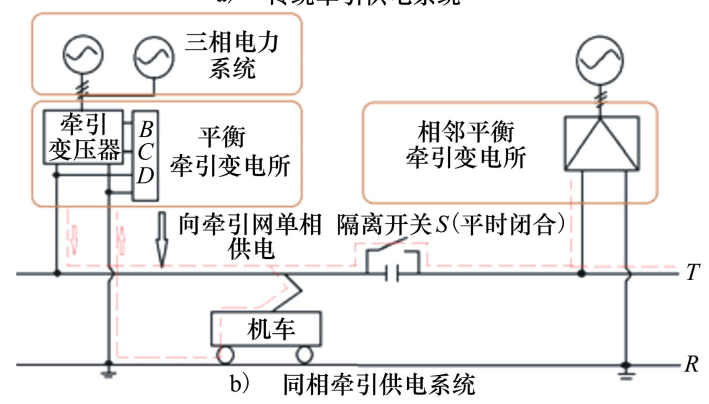

图 1 牵引供电系统结构图 
同相供电装置核心模块一般由 “背靠背” 对称 形式的单相桥式变流器构成。出于拓扑优化和经济 效益方面的权衡考量, 本文选用单相全桥结构的 PWM 整流电路作为同相供电装置的基本功能模块。 将平衡变换装置 BCD (balance convert device) 与平 衡接线变压器进行有机结合, 可构造出具有负序消 除及谐波抑制功能的同相供电装置。Scott 变压器 与其他常见平衡接线变压器的主要性能参数对比见 表 1(在变电所两臂负荷相同情况下)。由于 Scott 变压器具有容量利用率高、工作原理简单以及应用 广泛等优点,故将其作为牵引变压器的选型。

\section{表 1 各平衡接线变压器主要性能参数}

\begin{tabular}{ccc}
\hline 变压器类型参数 & $\begin{array}{c}\text { 最大容量 } \\
\text { 利用率 } / \%\end{array}$ & $\begin{array}{c}\text { 负序电流 } \\
\text { 不对称度 } / \%\end{array}$ \\
\hline Scott & 92.8 & 0 \\
$\mathrm{~V}, \mathrm{v}$ & 100 & 50 \\
$\mathrm{YN}, \mathrm{d} 11$ & 75.6 & 50 \\
\hline
\end{tabular}

\section{2 同相供电功能的实现机理}

Scott 平衡接线变压器实现的是 $a, b, c \rightarrow o, \alpha, \beta$ 的三相/两相系统变换。如图 2a) 所示, Scott 牵引变 压器的 $M$ 座副边绕组输出端直接与牵引网相连, $T$ 座副边绕组输出端接同相供电装置, 经同相供电装 置的无功、谐波补偿后, 再与 $M$ 座输出端并网, 共同 为牵引网及机车负载供 $50 \mathrm{~Hz}$ 单相交流电, 实现从 电力系统到接触网的三相/单相对称变换。以同相 供电装置为核心的平衡牵引变电所结构如图 $2 \mathrm{~b}$ ) 所示。

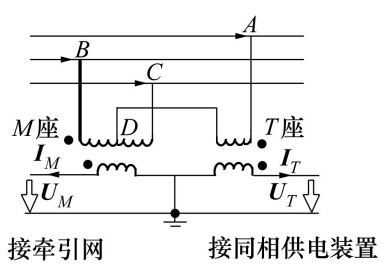

a) Scott平衡牵引变压器

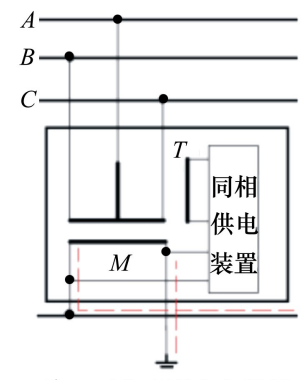

b) 平衡牵引变电器所
图 2 Scott 变压器结构及其在同相供电装置中的接线图

对图 2a) 中电网侧三相基波电流和负载电流进 行矢量关系分析可得两者之间的关系为

$$
\left\{\begin{array}{l}
I_{A}=2 K I_{T} \\
I_{B}=-K I_{T}+\sqrt{3} K I_{M} \\
I_{C}=-K I_{T}-\sqrt{3} K I_{M}
\end{array}\right.
$$

式中, $K=\frac{n}{2 \sqrt{3} N}, N, n$ 分别为原、副边绕组市数。

由于平衡接线变压器可消除零序分量, 故按对 称分量法可将三相基波电流分解为正、负序分量 $I^{+} 、 I^{-}$( 以 $A$ 相为例)

$$
\left\{\begin{array}{l}
I_{A}^{+}=K\left(I_{T}+j I_{M}\right) \\
I_{A}^{-}=K\left(I_{T}-j I_{M}\right)
\end{array}\right.
$$

结合 $I_{T}$ 及 $I_{M}$ 的相位关系分析可得正、负序电流 $I^{+} 、 I^{-}$的幅值表达式如下

$$
\left\{\begin{array}{l}
I^{+}=K \sqrt{I_{T}^{2}+I_{M}^{2}+2 I_{T} I_{M} \cos \left(\phi_{2}-\phi_{1}\right)} \\
I^{-}=K \sqrt{I_{T}^{2}+I_{M}^{2}-2 I_{T} I_{M} \cos \left(\phi_{2}-\phi_{1}\right)}
\end{array}\right.
$$

综上可得表征负序含量的电流不对称系数表达 式为

$$
\gamma=\frac{I^{-}}{I^{+}}=\sqrt{\frac{I_{T}^{2}+I_{M}^{2}-2 I_{T} I_{M} \cos \left(\phi_{2}-\phi_{1}\right)}{I_{T}^{2}+I_{M}^{2}+2 I_{T} I_{M} \cos \left(\phi_{2}-\phi_{1}\right)}}
$$

由 (4) 式可以看出, 要达到消除负序 $(\gamma=0)$, 同时降低谐波含量 (功率因数 $\cos \phi=1$ ) 的预定目 标, 实现同相贯通供电, 必须满足如下条件

$$
\left\{\begin{array}{l}
\phi_{T}=\phi_{M}=0 \\
I_{T}=I_{M}
\end{array}\right.
$$

以 (5) 式所描述的条件为目标, 展开同相供电 装置的工作机理分析如下:

为满足电力机车在加速牵引、匀速惰行以及回 馈制动等不同运行工况下具有能量双向流动性的供 电、馈电需求, 同相供电装置由 “背靠背”式的对称 单相全桥电路构成, 拓扑如图 3a) 所示。

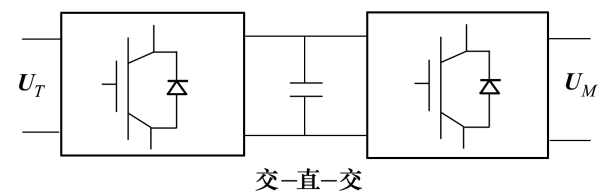

a) 单重同相供电装置拓朴

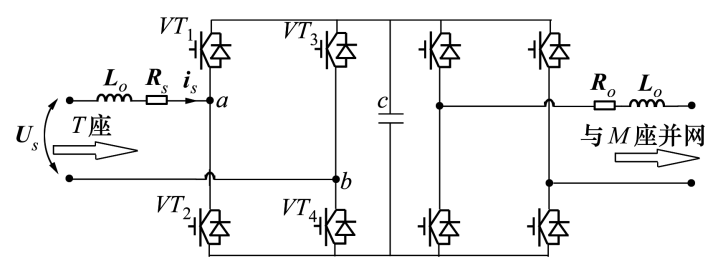

b) 结构原理图

图 3 单重同相供电装置 
结合元器件选型方法, 可搭建仿真电路如图 $3 b$ ) 所示。

以整流侧电路工作原理为例, 将图 3b) 中整流 电路输出侧视为等效电压源, 则根据 KVL 可归纳出 PWM 整流电路的等效电路数学表达式

$$
u_{L}=L \frac{\mathrm{d} i_{s}}{\mathrm{~d} t}=u_{s}-u_{a b}-R_{s} i_{s}
$$

结合系统感性负载特性可得如图 4 所示的电 压、电流矢量关系图。

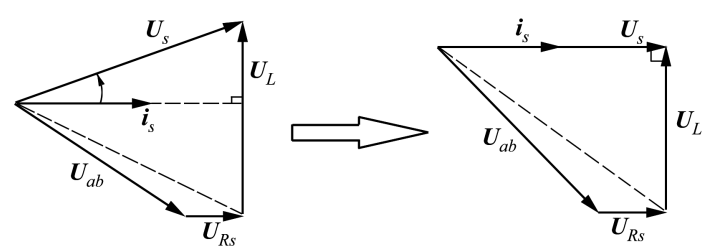

图 4 PWM 整流电路电压、电流同相控制原理图

图 4 表明, 同相供电装置通过对整流输人侧电 压 $u_{a b}$ 进行幅相控制, 进而可对高频输人电感电流 $i_{S}$ 做出适当调整, 最终实现网侧电压 $u_{S}$ 和电流 $i_{S}$ 同相 (即 $u_{T}$ 与 $i_{T}$ 同相)。同理, 逆变侧电路通过类似的电 压、电流相位控制和无功补偿作用, 可实现负载端电 压 $u_{M}$ 与 $i_{M}$ 同相以及 $i_{T}$ 与 $i_{M}$ 的等效值同幅 ${ }^{[7]}$ 。通过 上述控制过程,便可达到 (5) 式的平衡条件。

上述单重同相供电方案虽然在理论上达到了单 相供电的预定目标, 且存在功率因数为 1 的可能性, 但实际运行的机车负载具有非线性、波动性和随机 性, 仅靠上述单重装置并不能在现实应用中达到全 线贯通供电和电能质量最优的预定目标, 线路上仍 会保留中性分相区 ${ }^{[13]}$ 。综上可知, 单重同相供电装 置无论在内部控制算法还是整体拓扑结构方面都还 存在着很大的改进空间, 故在此基础上仍可做出创 新改进, 融合多类结构及控制算法优化方法, 得到性 能更优, 适用性和鲁棒性更强的新型四重化同相牵 引供电方案。

\section{2 四重化同相供电装置结构改良}

以多重化逆变电路为例, 利用各重电路依次错 相的原理,通过匹配变压器将具有相同或相似结构 的逆变电路耦合联结为一个整体, 进而借助各错相 电路输出波形的特定叠加与抵消作用, 可消除主要 的高次谐波, 实现整体电路电能质量改善的功能。 除此之外, 多重化技术还可大幅降低大容量开关器
件的工作频率, 提高输出电压等级, 这为该型电路的 大规模普及提供了前提 ${ }^{[11-13]}$ 。故本文引人成熟的 多重化变流技术与现有系统进行深人融合, 进一步 实现同相汼引供电系统的电能质量改善。

大容量逆变器可分为多重化逆变和多电平逆变 2 类, 其中多重化逆变按匹配变压器连接方式又可 分为串联型和并联型 ${ }^{[11]} 2$ 种, 前者主要用于大容量 APF, 后者主要用于大容量 STATCOM 和 UPFC 系 统中。综合考量后, 本文选用并联型多重化连接方 式。在重数选择方面, 过高的重数会增加同相供电 装置的结构复杂程度和投资成本; 但重数过低又会 降低装置的电能质量改善效能。故经过多型电路的 试验权衡对比, 本文最终选择四重化结构作为新型 多重化同相供电装置的结构重数。

按图 5 所示的多重化拓扑将 4 组结构完全相同 的单重同相供电装置叠加起来, 通过升、降压匹配变 压器连接为一个整体 (匹配变压器一方面用于降低 开关管端电压, 另一方面起到了隔离干扰的作用)。

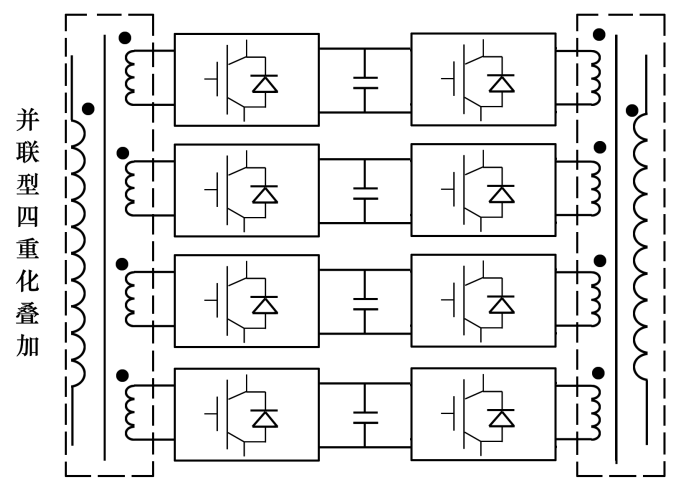

图 5 四重化同相供电装置结构拓扑图

根据多重化逆变单元的移相叠加原理, 为实现 最优的高次谐波补偿效果, 各重逆变电路应依次间 隔 $\pi / N$ 相角 $(N=3 m, m$ 表示多重化电路的重数)。 多重化逆变原理可参考如下波形示意图, 其中图 6a) 表示各依次错相的单重化 PWM 逆变电路输出 电压波形, 图 6b) 表示经移相叠加作用形成的四重 化逆变电路的 PWM 逆变输出电压波形。

参考图 6 所示的叠加过程, 各基本单元电路的 相位差会在输出叠加过程中发挥关键的抵消作用, 从而消除特定的高次谐波 $(3,5,7$ 次为主要高次谐 波), 使最终输出波形更近似于正弦基波, 大幅降低 了同相供电装置输出波形的谐波含量, 进一步改善 了牵引供电系统的电能质量。故将图 5 所示的四重 

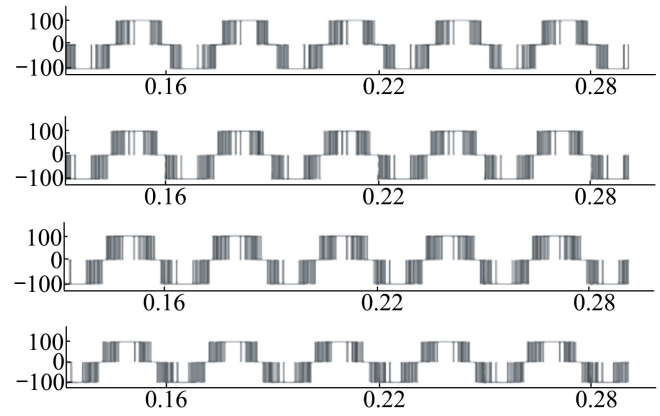

a) 依次间隔 $\pi / N$ 相角的单重化逆变电路输出电压波形

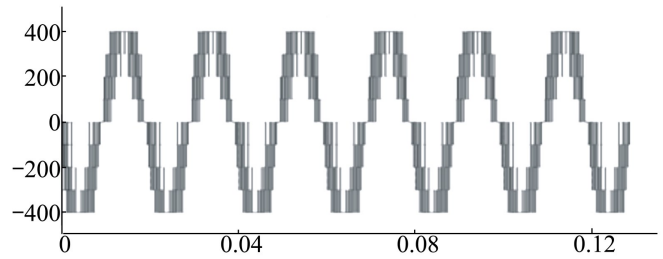

b）四重化 PWM 逆变电路输出电压波形

图 6 多重化逆变电路移相叠加原理

化叠加构想应用于平衡牵引变电所, 参考图 $1 \mathrm{~b}$ ) 和 图 $2 b)$, 得到集三相电力系统、同相牵引变电所、牵 引网及机车负载的完整牵引供电系统拓扑如图 7 所示。

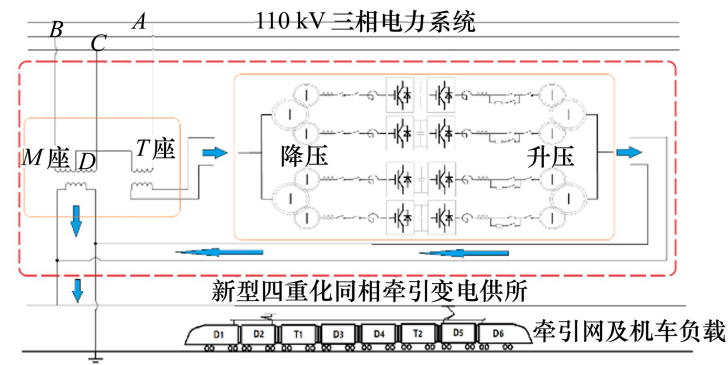

图 7 基于潮流控制技术的新型四重化 同相牵引供电系统结构示意图

此节在单重同相供电装置的基础上,通过变压 器及电路结构选型、引人多重化逆变技术等措施, 从 牵引变电所结构优化的角度改进得到了电能质量改 善性能更为优异, 适用性更为宽泛的新型四重化结 构。在满足变电所电能质量达标的前提下, 可将处 于同一供电区段的牵引变电所之间的分区所相连, 以进一步减少电分相, 实现铁路沿线的全线贯通供 电。下面, 将从四重化同相供电装置的控制算法优 化角度阐述新型系统的仿真模型搭建过程。

\section{3 控制算法优化及仿真模型搭建}

庞棋峰等 ${ }^{[7]}$ 提出一种单重同相供电装置的控 制算法,但实现该算法借助了标准正弦表、指令电流 相位控制表,这类通过实时查表形式来获取给定参 量的控制方法, 其控制精度易受参数准确度的影响, 响应速度受限于控制器的实时运算能力, 且不能适 应系统对于复杂工况的鲁棒性要求。王荣华等 ${ }^{[9]}$ 提出了统一功率控制的多重化设想,但该方案的控 制算法仅采用了基于幅相控制的简易开环算法,对 于机车负载的不良特性及复杂工况, 这类忽略系统 稳定性的开环控制算法显然不具有实际应用前景。 故本文针对上述系统存在的控制算法缺陷, 提出了 新型四重化同相供电装置的改进控制算法。

在本装置中, 各基本单元电路的结构具有对称 性,故它们各自的整流及逆变控制算法类似,唯一的 区别仅在于逆变环节中 PWM 控制信号产生模块采 用了依次错相 $\pi / 12$ (即 $\pi / 3 m$ ) 相角的高频载波。 故只用对某一单元电路的控制策略做出优化改进, 其他同理即可。

\section{1 整流及逆变电路控制算法优化}

PWM 整流电路的控制策略可分为直接电流控 制和间接电流控制两大类。通过控制 $\mathrm{H}$ 桥交流侧 电压来达到控制交流侧电流目的的间接电流控制方 法动态响应慢, 且对电路参数波动敏感, 并不适用于 控制精度要求高的场合。故本文在直接电流 PI 控 制方案 ${ }^{[7]}$ 的基础上, 使用归一化的 PLL (phase locking loop) 锁相电路代替查表式的数字 PLL 锁相 模块, 设计出系统响应性能更优的 PWM 整流电路 控制算法,其控制策略如图 8a) 所示。

由控制框图可见, 装置整流侧电路通过对输出 直流电压的反馈控制，进而实现对输人侧交流电压 $u_{S}$ 及高频输人电感电流 $i_{S}$ 的幅、相调节, 同时为消除 电网电压对馈网电流的影响, 特地引人了电压前馈 控制。引人归一化 PLL 锁相环模块, 在最终实现网 侧电压 $u_{S}$ 和电流 $i_{S}$ 同相 (即 $u_{T}$ 与 $i_{T}$ 同相) 的基础 上,还增强了控制算法的应用灵活性。

逆变电路同样采用了双闭环的控制策略, 受整 流电路控制策略的启发, 在输出电流、电压反馈控制 过程中,使用了归一化的参数处理算法,避免了不同 物理量纲及不同参数量级对 PI 控制器输人参量的 计算精度影响。逆变电路控制策略如图 8b) 所示。 


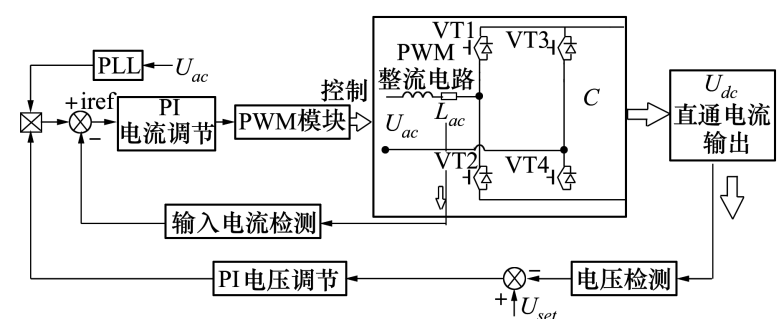

a) 整流电路控制策略优化框

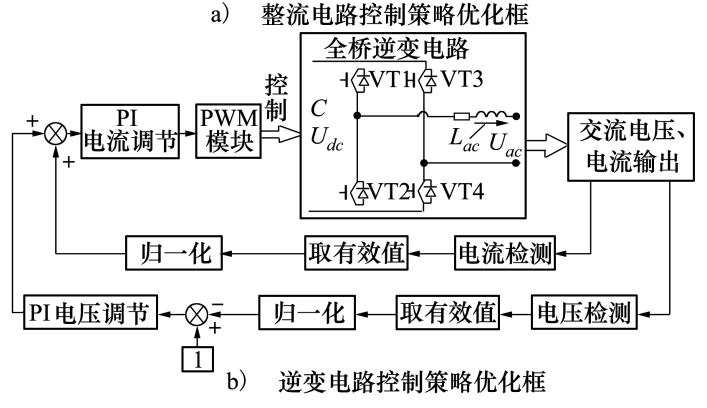

图 8 同相供电装置整流、逆变控制策略优化框图

由图 $8 \mathrm{~b}$ ) 控制策略分析可知, 逆变电路通过调 节负载电流来影响同相供电装置的输人功率, 进而 调节输人电流的幅值, 实现负载端电流的等幅调控 ( 即 $I_{T}=I_{M}$ ), 并通过负载侧的双闭环反馈调制, 来实 现有功传输和无功补偿, 最终使并网电压与电流同 相 (即 $u_{T}$ 与 $i_{T}$ 同相)。

除上述提及的控制算法优化措施之外,本四重 化装置还在整流与逆变电路控制算法中使用了倍频 PWM 技术, 该方法能成倍提高开关管的等效工作频 率, 克服大容量 IGBT 工作频率有限的缺陷。

\section{2 四重化同相供电装置的仿真电路搭建}

将前述优化算法应用于整流、逆变控制电路,结 合前面对平衡汼引变电所的结构改良设计, 并参照 某实际工程线路关键电气参数设计标准( 见表 2), 借助 MATLAB/Simulink 软件仿真平台, 成功搭建出 含四重化同相供电装置的平衡牵引变电所仿真电路 如图 9 所示。

\section{表 2 同相供电装置关键电气参量}

\begin{tabular}{cc||cc}
\hline 参数 & 数值 & 参数 & 数值 \\
$\begin{array}{c}\text { 电源短路 } \\
\text { 容量 } / \mathrm{MVA}\end{array}$ & 2253 & $\begin{array}{c}\text { 整流输人电压 } \\
\text { 三相额定 }\end{array}$ & $1000 / 1500$ \\
$\begin{array}{c}\text { 电压 } / \mathrm{kV} \\
\text { (V)/电流 }(\mathrm{A})\end{array}$ & 110 & $\begin{array}{c}\text { 输人电压 } / \mathrm{kV} \\
\text { 支撑电容 } / \mu \mathrm{F}\end{array}$ & 25.8 \\
输出电压 $/ \mathrm{kV}$ & 27.5 & $\begin{array}{c}\text { 直流电压 } / \mathrm{V} \\
\text { 逆变输出 } \\
\text { 电压 } / \text { 电流 }\end{array}$ & $1000 / 1500$ \\
输人频率 $/ \mathrm{Hz}$ & 50 & $\begin{array}{c}\text { IGT 开关 } \\
\text { 频率 } / \mathrm{Hz}\end{array}$ & 450 \\
\hline
\end{tabular}

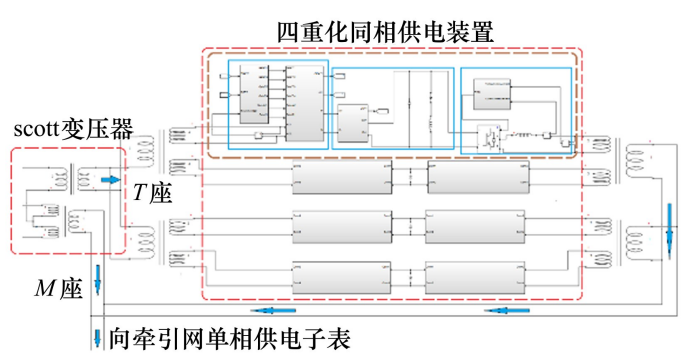

图 9 平衡牵引变电所仿真电路图

为简化模型表示, 将三个功能电路 (整流、直流 支撑和逆变) 进行子模块封装, 即可得到四个结构 完全相同的模块电路。最终, 借助匹配变压器并联 四个子模块的同时, 将其与 Scott 牵引变压器巧妙结 合, 通过载波移相控制技术成功构造出具有同相供 电功能, 且电能质量改善性能更优的新型四重化同 相牵引变电所仿真电路模型。

\section{3 考虑牵引网及机车负载的模型改进}

针对当前 “简化机车负载模型” 研究的局限性, 本文立足于完整牵引供电系统的全局角度来研究新 型同相供电装置的实际效能, 并以此为目标参照某 实际线路牵引供电系统的组成模块, 如图 10a), 成 功搭建出集三相电力系统、平衡牵引变电所、全并联 AT ( Auto-Transformer) 牵引网和 CRH6 ( China Railway High-Speed 6) 型动车组负载为一体的完整 同相牵引供电系统仿真电路, 如图 10b) 所示。

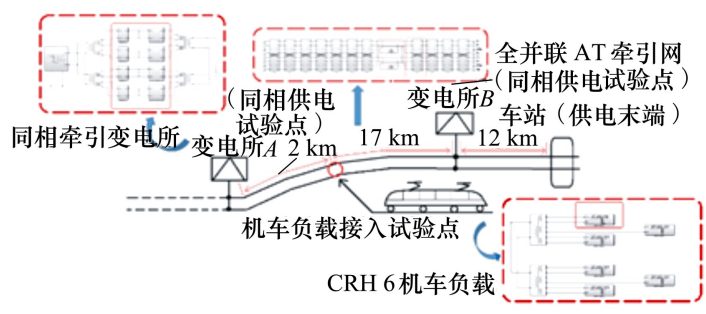

a) 实际线路牵引供电系统组成示意图

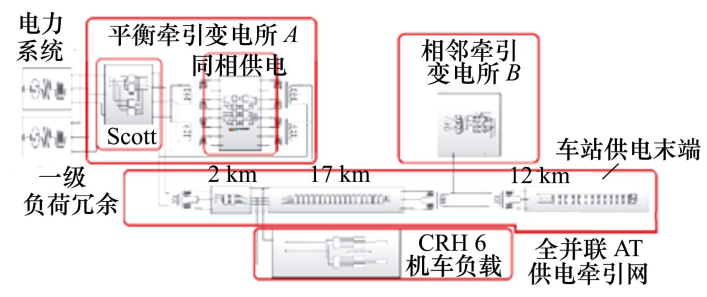

b) 带牵引网及机车负载的新型同相牵引供电系统仿真主电路

图 10 同相牵引供电系统带载模型

其中, 全并联 AT 汼引网电路中各传输线单位 阻抗 $(\Omega / \mathrm{km})$ 如下: 承力索 $\mathrm{J}$ 为 0.181 、接触线 $\mathrm{C}$ 为 
0.119 、单条钢轨 $R$ 为 0.135 、保护线 $P W$ 为 0.286 、架 空回流线 $\mathrm{F}$ 为 0.163 ; CRH6 动车组负载采用 $6 \mathrm{D} 2 \mathrm{~T}$ 的编组方式, 运用架控模式控制 $4 \times 6$ 台额定功率为 $400 \mathrm{~kW}$ 的三相交流异步电机, 电机采用 DTC 直接转 矩控制方式。限于篇幅, 此处不再赘述 AT 牵引网 (标准电压等级: $27.5 \mathrm{kV}$ ) 及 CTH6 动车组负载的工 作机理及模型搭建过程, 具体原理可参考相关文献 [14-15]。

在成功搭建含系统负载的改进模型基础上, 可 对 (5) 式表示的同相供电预定目标及电能质量优化 性能进行仿真试验与结果分析如下。

\section{4 试验验证}

本文借助 MATLAB/Simulink 软件仿真平台, 从 电路输出波形幅相分析和频谱分析两个方面综合验 证新型四重化同相供电装置的同相供电功能及其电 能质量改善效果, 并通过单重与四重化同相供电装 置的性能对比, 论证新型同相供电方案的性能优 越性。

\section{1 电压、电流输出波形幅相分析}

由前文总结的负序消除及功率因数最优条件可 知, 只要能达到 (5) 式所表示的三个条件 (即 $u_{T}$ 与 $i_{T}$ 同相、 $u_{M}$ 与 $i_{M}$ 同相、 $I_{T}=I_{M}$ ), 就可在理论上实现同相 供电, 同时将功率因数提高到 1 。故下面将通过仿 真输出波形来验证新型系统的同相供电效能。

在 MATLAB/Simulink 中以 $0.00001 \mathrm{~s}$ 的采样时 间对不包含同相供电装置的 Scott 变电所电路进行 仿真, 由示波器得到 $u_{M}, i_{M}$ 以及 $u_{T}, i_{T}$ 的波形如图 11a）至 11b) 所示。

a)

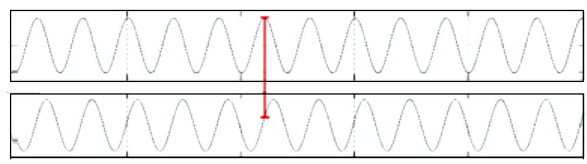

非平衡牵引变电所中 $M$ 座副边绕组输出电压、电流波形

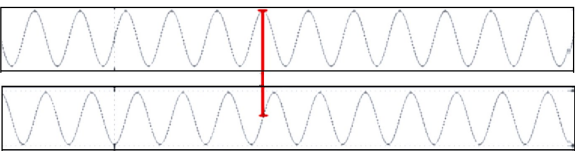

b）非平衡牵引变电所中 $T$ 座副边绕组输出电压、电流波形

图 11 非平衡条件下 Scott 变压器输出电压、电流波形

分析电压、电流波形图中的相位对比辅助标识 可知,在不含同相供电装置的非平衡牵引变电所中, 由于机车负载的阻感特性, 故电压均超前于电流一
定相位, 即 $u_{T}$ 与 $i_{T}, u_{M}$ 与 $i_{M}$ 均不同相, 不满足并网条 件, 牵引供电系统只能采用含电分相区的异相轮换 供电制。(为方便电压、电流的相位观察, 此处仅在 系统带等效阻感负载情况下仿真即可, 不影响调相 功能的验证) 采用同相供电技术后, 平衡汼引变电 所电路仿真输出波形如图 12a) 至 $12 b$ ) 所示。

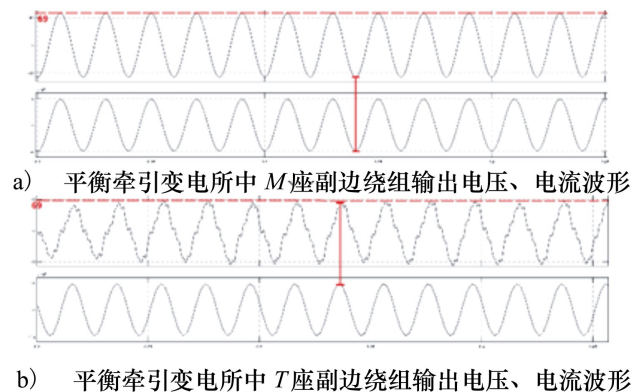

图 12 平衡条件下 Scott 变压器输出电压、电流波形

由上面波形可知,加人同相供电装置后, 通过其 负序消除和无功、谐波补偿作用, 在牵引网及机车负 载扰动误差允许范围内, 达到了 $u_{M}$ 与 $i_{M}$ 同相、 $u_{T}$ 与 $i_{T}$ 同相以及 $i_{T}$ 与 $i_{M}$ 同幅 (即有效值在误差允许范围 内相等 $\left.I_{T}=I_{M}\right)$ 的预定目标。

上述实验结果表明,本文在单重同相供电装置 基础上所做的结构改良和算法优化措施, 不影响同 相供电装置实现电压、电流幅相调控及功率因数改 善的功能。故在此前提下, 可借助 FFT( Fast Fourier Transform) 的频谱分析方法, 分别对单重和四重化 带载同相供电装置进行仿真对比分析, 验证新型系 统具有更优的电能质量改善性能。

\section{2 基于快速傅里叶变换的频谱分析}

按同相供电装置的结构及控制原理可搭建单重 仿真电路, 图略, 可参考图 9 中的单重子模块。

对上述单重同相供电电路进行带载仿真运行, 可得 SPWM 逆变电路的输出电压、电流波形如图 13a) 所示。

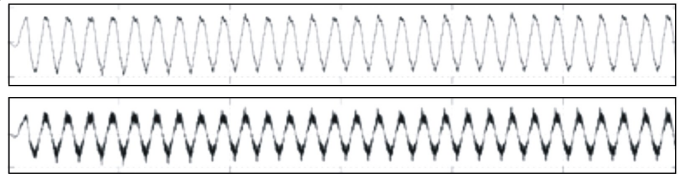

a）单重同相供电装置输出电压、电流波形
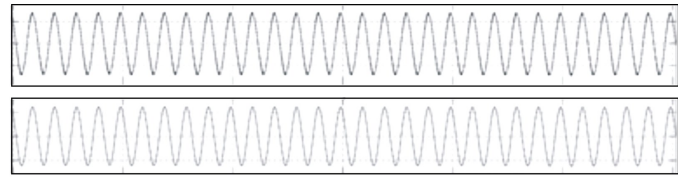

b）四重化同相供电装置输出电压、电流波形

图 13 同相供电装置输出电压、电流波形 
在 FFT 频谱分析参数设置时, 为防止波形畸变 严重, 要求信号的带宽通常略小于奈奎斯特频率 (Nyquist frequency), 故取奈奎斯特频率作为计算的 最大频率; 此外, 为了得到较为可靠的分析结果, 特 在波形稳定时段内 (约 $1.1 \mathrm{~s}$ 之后) 取 2 个有效周期 进行快速傅里叶分析; 一般情况下 $1000 \mathrm{~Hz}$ 处的总 谐波失真最小, 故取最大频率为 $1000 \mathrm{~Hz}_{\circ}$ 将图 13a) 所示的示波器数据缓存, 做快速傅里叶分析可 得如下的电压频谱图。

从图 14 的频谱分析结果可以看出, 单重同相供 电装置的输出电压总谐波畸变率 THD (total harmonic distortion) 大约为 $12.36 \%$, 即电路功放时, 输出信号与输人信号相比, 增加了 $12.36 \%$ 的谐波成 分。同理还可得出输出电流 THD 值约为 $12.08 \%$ (图略)。

从上述频谱分析结果可以看出, 虽然单重结构 的同相供电装置能够在消除负序的基础上, 得到谐 波含量较少的类正弦电压、电流波, 但其 THD 值均 超过了 $12 \%$, 故仍有较大的电能质量提升空间。

对新型四重化同相供电装置进行带载仿真运 行,可得如下所示的输出电压、电流波形。
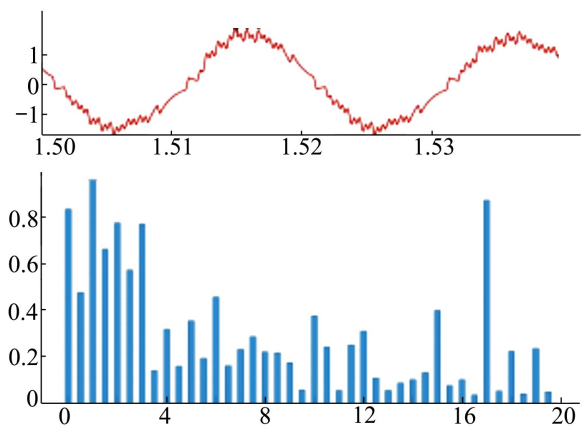

图 14 单重同相供电装置输出电压 FFT 频谱图

将图 13a) 至 13b) 的波形进行对比分析可以看 出, 进行四重化结构改进和控制算法优化后, 同相供 电装置的输出波形变成了更为平滑的正弦波。同样 对其做 FFT 频谱分析可得如下所示的频谱图 (频谱 分析参数设置同上）。

由图 15 可知, 四重化同相供电装置的输出电压 THD 值约为 $5.17 \%$, 输出电流 THD 值约为 $2.10 \%$ 。 综上可得单重同相供电装置与改良的新型四重化同 相供电装置的输出信号频谱分析结果对比如下:
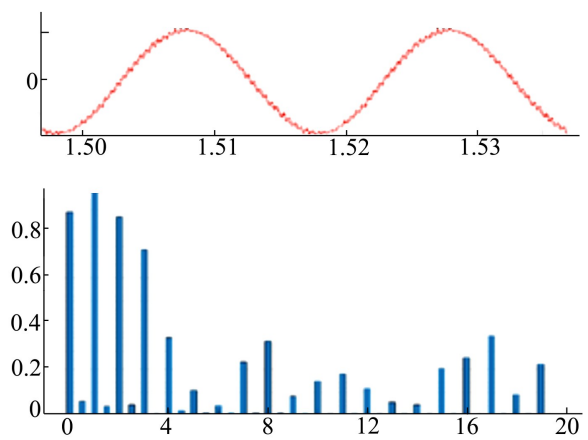

a）四重化同个供电装置输出电压 FFT 频谱图
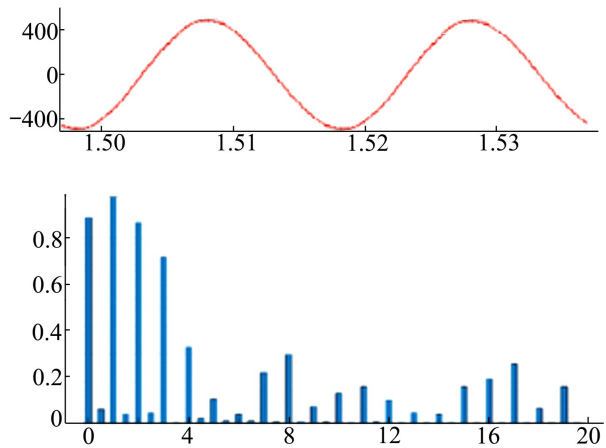

b）四重化同个供电装置输出电流 FFT 频谱图

图 15 四重化同相供电装置输出信号 FFT 频谱分析

表 3 同相供电装置结构多重化改良效果

\begin{tabular}{cccc}
\hline THD 值 & 单重结构 & 四重化结构 & 谐波含量减少 \\
\hline 输出电压 & $12.36 \%$ & $5.17 \%$ & $66.26 \%$ \\
输出电流 & $12.08 \%$ & $2.10 \%$ & $82.62 \%$ \\
\hline
\end{tabular}

由表 3 数据对比分析可以看出, 本文提出的新 型四重化同相供电装置在带载高仿真度的情况下, 可将其输出信号中的谐波含量减少 6 层以上, 其中 对电流信号的改善效果最优, 达到了 $82.62 \%$ 的优 化度。

上述信号波形及频谱分析结果表明,本文提出 的新型四重化同相供电装置在实现负序消除, 进而 取消变电所 “电分相区” 的同时; 还在原单重同相供 电装置实现干扰隔离的基础上, 进一步提升了其改 善电能质量的效能。实验结果的各项指标均表明本 文对单重同相供电装置提出的结构改进和控制算法 优化方案具有极大的实际应用价值和社会经济 效益。 


\section{5 结 论}

针对传统异相牵引供电系统及单重同相供电技 术的现有缺陷, 本文提出一种基于潮流控制技术的 新型四重化同相牵引供电方案, 对现有的牵引供电 技术领域进行了完善与创新。具体工作如下:

1) 在深人分析异相供电与单重化同相供电装 置的缺陷基础上, 采用载波移相技术对同相供电装 置进行结构改良, 提出四重化并联结构;

2) 针对单重同相供电方案存在巨大的电能质 量提升空间的现状,通过有效融合倍频 PWM 技术、 归一化的 PLL 锁相技术以及 PI 控制器参数优化等 技术,实现装置的控制算法优化;

3）针对现有 “简易负载模型” 的研究局限性, 提出包含汼引网及机车负载的完整牵引供电系统 模型;

4) 基于 MATLAB/Simulink 软件仿真试验平 台,对本文所提的优化方案进行可行性对比实验 验证。

试验结果的时域及频域综合分析结果表明, 本 文提出的新型四重化同相供电方案不仅具有良好的 负序消除功能, 还拥有明显优于单重结构的电能质 量改善性能, 这充分体现出本新型牵引供电方案具 有极高的实用参考价值和巨大的发展潜力。

同时也应认识到四重化结构所带来的影响有:

1) 复杂的电路结构成倍地增加了元器件损耗 量, 提高了装置的初期投人成本。

2) 从电力器件故障诊断的角度来看, 开关管、 电容器等器件数量的成倍提高, 增大了装置的总故 障率( 载波依次移相的单元电路并不具备良好的圥 余特性)。

如何寻找更优的元器件替换、改进系统的容错 控制, 成为了本文未来的研究方向。

\section{参考文献:}

[1] 李群湛. 我国高速铁路牵引供电发展的若干关键技术问题 [J]. 铁道学报, 2010,32(4) : 119-124

LI Qunzhan. On Some Technical Key Problems in the Development of Traction Power Supply System for High-Speed Railway in China[J]. Journal of Railways, 2010, 32(4) : 119-124 (in Chinese)

[2] 张志春, 吴丽然. 基于 $V_{v}$ 或 Scott 汼引变压器的同相供电系统及其控制 $[\mathrm{J}]$. 控制与信息技术, 2018 (4) : 32-35

ZHANG Zhichun, WU Lilan. Co-Phase Traction Supply System Based on Vv or Scott Traction Transformer and Its Control $[\mathrm{J}]$. Control and Information Technology, 2018(4) : 32-35 (in Chinese)

[3] 曾国宏,郝荣泰. 基于有源滤波器和斯科特变压器的同相牵引供电系统 $[\mathrm{J}]$. 北方交通大学学报, 2003(4) : 84-90

ZENG Guohong, HAO Rongtai. Co-Phase Traction Supply System Based on Active Power Filter and Scott Transformer[J]. Journal of Northern Jiaotong University, 2003(4) : 84-90 (in Chinese)

[4] 张秀峰, 钱清泉, 李群湛, 等. 基于有源滤波器和 AT 供电方式的新型同相牵引供电系统 $[\mathrm{J}]$. 中国铁道科学, 2006(6): 73-78

ZHANG Xiufeng, QIAN Qingquan, LI Qunzhan, et al. A Novel Co-Phase Power Supply System Based on Active Power Filter and AT Power Supply Mode[J]. China Railway Science, 2006(6) : 73-78 (in Chinese)

[5] 彭露. 牵引变电所无功补偿方案优化与实践 $[\mathrm{D}]$. 上海:华东交通大学, 2017

PENG Lu. Optimization and Practice of Reactive Power Compensation Scheme for Traction Substation[D]. Shanghai : East China Jiaotong University, 2017 (in Chinese)

[6] 杨博. 同相供电装置主电路研究 $[\mathrm{D}]$. 成都: 西南交通大学, 2010

YANG Bo. Research on Main Circuit of Co-Phase Traction Power Supply System Device[D]. Chengdu: Southwest Jiaotong University, 2010 (in Chinese)

[7] 庞棋峰. 电气化铁路同相供电潮流控制实验装置研究与设计 [D]. 成都: 西南交通大学, 2012

PANG Qifeng. A Study and Design on The Power Flow Controller Experiment Device for Co-Phase Traction Power Supply System [D]. Chengdu: Southwest Jiaotong University, 2012 (in Chinese)

[8] 刘婷婷. 基于直流背靠背输电技术的同相牵引供电系统研究 $[D]$. 大连: 大连交通大学, 2013

LIU Tingting. Co-Ohase Traction Power Supply System Based on Back-to-back HVDC Transmission Technology [D]. Dalian:

Dalian Jiaotong University, 2013 (in Chinese) 
[9］王荣华,文纲. 基于统一功率控制的牟引供电系统补偿装置 $[\mathrm{J}]$. 控制与信息技术, 2018(1): 52-56

WANG Ronghua, WEN Gang. Compensation Facility for Traction Power System Based on Unified Power Manipulating $[\mathrm{J}]$. Control and Information Technology, 2018(1): 52-56 (in Chinese)

[10] 宋平岗, 周振邦, 董辉. 新型的铁路电能质量综合治理控制策略 $[\mathrm{J}]$. 电力系统及其自动化学报, 2018,30(8): 93-101

SONG Pinggang, ZHOU Zhenbang, DONG Hui. Novel Control Strategy for Integrated Management of Railway Power Quality $[\mathrm{J}]$. Journal of Power System and Automation, 2012,30(8) : 93-101 (in Chinese)

[11] 窦祥, 徐宁博, 孙德. H 桥逆变电路的并联移相 SPWM 多重化技术研究 $[\mathrm{J}]$. 国外电子测量技术, 2018,37(8): 52-56 DOU Xiang, XU Ningbo, SUN De. Research on Parallel Phase Shifted SPWM Multiplex Technology for H-Bridge Inverter Circuit [J]. Foreign Electronic Measurement Technology, 2016,37(8): 52-56 (in Chinese)

[12] 周洪霞,李洪烈,任剑. PWM 型逆变器多重化的仿真研究 $[J]$. 信息技术, 2014(5): 98-100

ZHOU Hongxia, LI Honglie, REN Jian. Multiple Configuration of PWM Inverter and Its Simulation [J]. Information Technology, 2014( 5 ) : 98-100 (in Chinese)

[13] 杨皓,熊成林, 吴瑕杰, 等. 组合式虚拟同相柔性供电装置的直接功率控制 [ J ]. 电网技术, 2019,43(4)：1196-1201

YANG Hao, XIONG Chenglin, WU Xiajie, et al. Direct Power Control of Combined Virtual Co-Phase Power Supply System[J]. Power Grid Technology, 2019, 43(4) : 1196-1201 (in Chinese)

[14] 郭晓旭. 高速铁路牵引网建模与仿真 $[\mathrm{D}]$. 成都:西南交通大学, 2014

GUO Xiaoxu. Modeling and Simulation of Traction Network of High-Speed Railway [D]. Chengdu: Southwest Jiaotong University, 2014

[15] 何丹炉,梁君海, 丁参参. CRH6F 型城际动车组研制 $[\mathrm{J}]$. 铁道车辆, 2014,52(12):14-17

HE Danlu, LIANG Junhai, DING Sansan. Development of CRH6F Intercity Multiple Units[J]. Railway Rolling Stock, 2014, 52 (12) : 14-17 (in Chinese)

\title{
Improvement of Quadruple Structure and Optimization of Control Algorithm for Co-Phase Traction Power Supply System
}

\author{
CHENG Shu, LIU Chang, TANG Jianxiang, YU Tianjian, LI Kaidi \\ (School of Transportation Engineering, Central South University, Changsha 417000, China)
}

\begin{abstract}
Co-phase power supply is one of the key technologies to solve the technical bottlenecks such as electrical phase separation zone, poor power quality and defects in structure and control algorithms in traditional traction power supply systems and single co-phase power supply schemes, and an inevitable way to realize the development of electrified railways in the direction of safety, high speed and heavy load. Based on the single co-phase power supply technology, a novel quadruple co-phase power supply scheme with negative sequence elimination and to suppress reactive power and harmonic better for the two aspects of the system structure improvement and control algorithm optimization is proposed by combining the technologies of power system flexible transmission grid-connected and multiple inverters. Finally, the simulation model for the novel co-phase traction power supply system was designed and built, and the present method was verified by a set of simulation experiments so as to obtain the expected results.
\end{abstract}

Keywords : co-phase traction power supply; multiple inverter; power flow control; power quality 